\title{
DYNAMO DRIVEN MEAN MAGNETIC FIELD IN ACCRETION DISKS OF COMPACT ASTROPHYSICAL OBJECTS AND ITS MANIFESTATIONS
}

\author{
G.D. CHAGELISHVILI, R.G. CHANISHVILI, J.G. LOMINADZE, \\ Z.A. SOKHADZE \\ Abastumani Astrophysical Observatory \\ Academy of Sciences of Georgian SSR \\ Pavlov str. $2 a$ \\ 380060 Tbilisi, USSR
}

\begin{abstract}
The simplest case of the nonlinear turbulent dynamo mechanism is proposed. It is shown that under certain conditions the generated mean magnetic field can become stronger than the small-scale one. Some manifestations (the model of Cyg $\mathrm{X}-1$ bimodal behaviour, asymmetric accretion onto the magnetized rotating compact star) of this mean field are discussed.
\end{abstract}

It can be considered that the existence of mean magnetic fields in the majority of astrophysical objects is determined by the turbulent dynamo action where the field generation sources are the differential rotation of the medium and the gyrotropic character of the turbulence (Moffatt, 1978; Weinstein et al., 1980). In the accretion disks the convective turbulence is small-scaled $\left(\ell_{z} \approx 0.1 \mathrm{Z}_{0}\right)$ (Chagelishvili and Lominadze, 1984), and this fact, at the Kepler rotation, leads to the anomalous large values of dynamo number (D), the only parameter determining the mean field growth rate in the turbulent dynamo linear theory in $\alpha \omega$ approximation. The accretion time is long (Shakura and Sunyaev, 1973), anyway it is much longer than the mean field growth time. That is why the generated mean magnetic fields are capable to grow up to the maximum possible values. The determination of these values and the magnetic field structure requires the help of the strongly nonlinear theory where, in the simplest case, two more parameters $-\xi$ and $\vec{B}_{0}-\operatorname{appear}\left(\vec{B}_{0}=\left(B_{0 r}, B_{0 \phi}, B_{0 z}\right)\right.$ determines the value and structure of seed average magnetic field, and $\xi$ the order of mean field influence on the average turbulent helicity). The three parameters $D, \xi$ and $\vec{B}_{0}$ are of equal importance.

Considering the convectively active geometrically thin disk $\left(z_{0} * r\right.$ where $z_{0}$ is the disk half-width and $r$ the distance from the considered region up to the disk centre), we can write down the turbulent dynamo nonlinear equations in $\alpha \omega$ approximation (in the one-dimensional case)

$$
\frac{\partial B_{\phi}}{\partial t}=G B_{r}+\lambda \frac{\partial^{2} B_{\phi}}{\partial z^{2}}
$$

R. Beck et al. (eds.), Galactic and Intergalactic Magnetic Fields, 395-398. (C) 1990 IAU. Printed in the Netherlands. 


$$
\frac{\partial B_{r}}{\partial t}=-\frac{\partial}{\partial z}\left[\alpha(z, B) B_{\phi}\right]+\lambda \frac{\partial^{2} B_{r}}{\partial z^{2}}
$$

Here $\lambda$ is the turbulent magnetic diffusion coefficient, $G \equiv r(\partial \omega / \partial r)$ characterizes the medium differential rotation in the disk and $\alpha(z, B)$ denotes the nonlinear helicity. We use the expression for $\alpha$, obtained in Chagelishvili et al. (1986a)

$$
\alpha(z, B)=\alpha_{0} \psi(z) f(B)
$$

where

$$
f(B)=\frac{1}{1+\xi \frac{B^{2}}{4 \pi \rho V_{t}^{2}}} \approx \frac{1}{1+\xi \frac{B_{\phi}^{2}}{4 \pi \rho V_{t}^{2}}}
$$

$\rho \mathrm{V}_{t}{ }^{2}$ is the turbulent pulsation energy density, $\alpha_{0}=\left(\ell^{2} \omega / \mathrm{z}_{0}\right)$ the helicity measure in turbulent dynamo kinematical theory, $\ell$ the characteristic scale of turbulence, and $\psi(z)$ determines the helicity distribution across the disk. Besides, it is considered that $B_{z} * B_{r} * B_{\phi}$. In dimensionless variables:

$$
\begin{aligned}
& \frac{\partial B_{\phi}}{\partial t}=D B_{r}+\frac{\partial^{2} B_{\phi}}{\partial z^{2}} \\
& \frac{\partial B_{r}}{\partial t}=-\frac{\partial}{\partial z}\left[\frac{\psi(z)}{1+\xi B_{\phi}^{2}} B_{\phi}\right]+\frac{\partial^{2} B_{r}}{\partial z^{2}}
\end{aligned}
$$

Solution of equations (5) and (6) requires the introduction of the initial mean magnetic field $\vec{B}(z, t)$

$$
\left.B_{r}(z, t)\right|_{t=0} \equiv B_{r 0}(z) ;\left.\quad B_{\phi}(z, t)\right|_{t=0} \equiv B_{\phi 0}(z)
$$

Obviously there appear three parameters $D, \xi$ and $\vec{B}_{0}$ in the one-dimensional nonlinear $\alpha \omega$ dynamo theory. According to Chagelishvili et al. (1986a) the value of $\xi$ is close to 1 ,

$$
\xi \approx 0(1)
$$

At the linear stage of generation the fundamental harmonic of the quadrupole mode is mainly increased (Moffatt, 1978; Weinstein et al., 1980). That is why this mode will become dominant when the nonlinear regime is reached (if the absolute value of the seed field is small). Otherwise, the mean nonlinear magnetic field will include both quadrupole and dipole - components.

As the possible manifestation of mean magnetic field we can consider the bimodal accretion model for Cyg $\mathrm{X}-1$ (Chagelishvili et al., 1986b, 
1988). The model assumes a variation of the accretion rate $\dot{M}$ in a certain interval $\left(\dot{\mathrm{M}}_{1}, \dot{\mathrm{M}}_{2}\right)$ and the existence of some critical rate $\dot{\mathrm{M}}_{\mathrm{cr}}$ in it. The convective turbulence is only inherent to the inner, radiationdominated region of the accretion disk and is always absent in the outer region. Thermal convection is also absent in the middle region if $\dot{\mathrm{M}}>\dot{\mathrm{M}}_{\mathrm{cr}}$, but for $\dot{\mathrm{M}}<\dot{\mathrm{M}}_{\mathrm{cr}}$ the parameters of the region are such that it becomes unstable against thermal convection. This circumstance makes the accretion disk magneto-active and switches Cyg X-1 to the "low" state.

Originally the magnetic field is carried to the disk by the matter coming from the optical component of the binary system. A part of this large-scale field may be considered as a seed field for the processes described by the turbulent dynamo equations. In the outer region, where helical turbulence is absent, the mean field decreases due to turbulent diffusion. If thermal convection is still absent in the middle region $\left(\dot{M}>\dot{M}_{c r}\right)$, decrease of the transported mean field continues. In this case the matter is not able to supply the inner region of the disk with a sufficiently strong mean magnetic field and in spite of the fact that the mean magnetic field is generated in the inner region of the disk, as the estimations show, it does not have time to grow up to perceptible values, and accretion goes on without the mean field. Thus we can say that when $\dot{M}>\dot{M}_{c r}$, Cyg X -1 is in a "high" state.

When $\dot{\mathrm{M}}<\dot{\mathrm{M}}_{\mathrm{cr}}$ thermal convection appears in the middle region of the disk and the generation of the mean magnetic field begins long before the matter comes to the inner region. The generated mean magnetic field is virtually toroidal (Chagelishvili et al., 1986a). Under such conditions the magnetic forces become stronger in the region of main energy release and they have a real influence on the matter dynamics. Namely, they can give rise to Parker instability.

Another example of mean magnetic field manifestation is the asymmetric disk accretion onto magnetized rotating compact stars. We consider the case, when the Alfvén and the co-rotation radii are close to each other: $R_{A} \simeq R_{C}$. In this case the existence of two magnetic fields of different origin - accretion disk toroidal field $\left(\vec{B}_{\phi}\right)$ and rotator dipole field $\left(\vec{B}_{D}\right)$ - leads to the occurrence of asymmetry, and this is determined by $\vec{B}_{\phi}$ and $\vec{B}_{D}$ mutual orientation: the manifestation of this asymmetry is that plasma will accrete mainly to one of the rotator magnetic poles. The reason for this asymmetry is the following: star and accretion disk magnetic fields are crossed. That promotes to their reconnection on the Alfvén surface and the latter leads to the formation of "channels", along them the accreting plasma "slips" mainly to one of the rotator magnetic poles. The "choice" of the magnetic pole depends on the $R_{C} / R_{A}$ ratio.

Finally, we enumerate those observational manifestations, to which the above discussed accretion can lead:

- If such a system is capable to generate jets, then they will be observed in only one direction;

- even the slightest change of accretion rate can lead to $\left(R_{C} / R_{A}\right)-1$ sign change. Taking into account that $R_{A}$ may be hundreds of times bigger than the star radius, this will significantly effect radiation of the given source directed towards the observer, and may become, for example, the explanation of the variability of some transients. 


\section{Acknowledgement}

We want to express our gratitude to Prof. P. Kronberg and Prof. R. Wielebinski for their help in publishing this paper.

\section{References}

Chagelishvili, G.D. and Lominadze, J.G. (1984) Proc. Course and Workshop Plasma Astrophysics, Varenna, Italy, ESA SP-207.

Chagelishvili, G.D., Lominadze, J.G. and Sokhadze, Z.A. (1986a) Proc. Joint Int. School and Workshop Plasma Astrophysics, Sukhumi, USSR, ESA SP-251, 523-529.

Chagelishvili, G.D., Chanishvili, R.G. and Lominadze, J.G. (1986b) Proc. Joint Int. Course and Workshop Plasma Astrophysics, Sukhumi, USSR, ESA SP-251, 563.

Chagelishvili, G.D., Chanishvili, R.G. and Lominadze, J.G. (1988) Adv. Space Res. $8(2), 217$.

Moffatt, H.K. (1978) Magnetic Field Generation in Electrically Conducting Fluids, Cambridge University Press.

Shakura, N.I. and Sunyaev, R.A. (1973) Astron. Astrophys. 24, 337-359.

Weinstein, S.I., Zel'dovich, Ya.B. and Ruzmaikin, A.A. (1980) Turbulent Dynamo in Astrophysics, NAUKA, Moscow.

MAKAROV: What is the parameter $\xi$, and is it significant?

SOKHADZE: The parameter $\xi$ determines the order of mean magnetic field influence on the average helicity of the turbulence. Besides, the determination of $\xi$ 's real meaning plays a significant role: the value of the observed averaged magnetic field can be proved only by the $\xi$ values close to 1 ( $\xi \approx 1$ on the order of magnitude), otherwise we have to deal with magnetic fields which are about three orders of magnitude less than the observed one.

ROSE: Please comment on the Cygnus $\mathrm{X}-1$ accretion disk.

SOKHADZE: Recent calculations that I have completed show that for the physical parameters generally assumed for the black hole Cygnus $\mathrm{X}-1$ (i.e. mass $\simeq 10 \mathrm{M} \odot$, mass accretion rate $\simeq 10^{-9} \mathrm{M}_{\odot} / \mathrm{yr}$ ) the inner region of the accretion disk is convective. This circumstance is likely to help to explain the high temperatures indicated by its observed $\mathrm{X}$-ray emission. 